J. Lake Sci. (湖泊科学) , 2016, 28(6): 1404-1410

DOI 10. 18307/2016. 0626

(c) 2016 by Journal of Lake Sciences

\title{
淡水磷监测中钼酸铵分光光度法的改进"
}

\author{
黄丰明, 唐鹊辉, 彭 亮, 肖利娟** \\ (暨南大学水生生物研究中心,广州 510632)
}

\begin{abstract}
摘 要: 我国现行淡水水体中磷测定方法为钿酸铵分光光度法 ( GB 11893-1989), 该方法检测下限为 $0.01 \mathrm{mg} / \mathrm{L}$, 但我国 南亚热带地区部分水体的总磷以及多数水体的溶解态磷浓度均低于 $0.01 \mathrm{mg} / \mathrm{L}$, 通过对标准试样和野外水样的测定, 分析 该方法在监测低磷浓度水体中可能出现的问题并提出改进方法. 结果表明:对于磷浓度低于 $0.01 \mathrm{mg} / \mathrm{L}$ 的水体,国标法灵 敏度低,准确性差. 通过 3 个方面的改进可提高该方法测定的灵敏度和准确性: 1) 将消解水样体积由 $25 \mathrm{ml}$ 增加到 $50 \mathrm{ml}$, 消解后不再进行二次稀释定容;2) 改变显色剂的配制, 显色剂由 $14 \%$ 硫酸溶液 $50 \mathrm{ml}$ 、酒石酸锺钾溶液 $5 \mathrm{ml}$ 、4\%钿酸铵溶 液 $15 \mathrm{ml}$ 和 $0.1 \mathrm{~mol} / \mathrm{L}$ 的抗坏血酸溶液 $30 \mathrm{ml}$ 混合而成,现配现用; 3 ) 将测定波长设置为 $880 \mathrm{~nm}$. 改进后方法的灵敏度有 较大提高, 能准确、有效地检测磷浓度在 $0.003 \sim 0.01 \mathrm{mg} / \mathrm{L}$ 的水样.
\end{abstract}

关键词: 钿酸铵分光光度法; 低磷; 淡水水体; 方法改进

\section{Improvement of the ammonium molybdate spectrophotometric method for phosphorus monitoring in freshwater}

\author{
HUANG Fengming, TANG Quehui, PENG Liang \& XIAO Lijuan ** \\ (Institute of Hydrobiology, Jinan University, Guangzhou 510632, P.R.China)
}

\begin{abstract}
Ammonium molybdate spectrophotometry ( GB 11893 - 1989) has been widely used to determine phosphorus for fresh waters, with a detection limit of $0.01 \mathrm{mg} / \mathrm{L}$. However, total phosphorus as well as dissolved phosphorus concentration in most fresh waters are lower than that in southern China. Here, we present an improvement of the method for monitoring low phosphorus concentrations in fresh waters after extensive testing of standard and field samples. The accuracy and sensitivity improved after changing three manipulations: 1) increase the digested sample volume from $25 \mathrm{ml}$ to $50 \mathrm{ml}$, without secondary dilution after digestion; 2) prepare the chromogenic agent as follows: mixing $50 \mathrm{ml}$ of $14 \% \mathrm{H}_{2} \mathrm{SO}_{4}, 5 \mathrm{ml}$ of antimony potassium tartrate solution, $15 \mathrm{ml}$ of ammonium molybdate solution and $30 \mathrm{ml}$ of ascorbic acid solution together just before addition; 3 ) set the determination wavelength to $880 \mathrm{~nm}$. The improved method is able to accurately determine phosphorus concentrations of $0.003-0.01 \mathrm{mg} / \mathrm{L}$.
\end{abstract}

Keywords: Ammonium molybdate spectrophotometry method; low phosphorus concentration; freshwater; improvement of method

磷是生物生长所必需的大量元素之一,多数淡水水体中的藻类生长主要受磷的限制 ${ }^{[1-2]}$,水体中磷浓度 的变化对浮游植物总量和群落结构有重要的影响 ${ }^{[3-4]}$. 当水体中溶解性磷浓度低于 $0.01 \mathrm{mg} / \mathrm{L}$ 时, 部分浮游 植物种类生长受到限制, 浓度越低受到限制的种类越多 ${ }^{[5]}$, 因此对水体中磷的监测是浮游植物群落生态、水 质管理和水生态学研究的基础内容. 目前对磷的测定方法有很多,包括分光光度法、原子光谱法和色谱法 等 ${ }^{[6]}$,其中钼酸铵分光光度法是测定水体中磷浓度最常见的方法,该方法因其操作简单、准确性高而被广泛 应用 ${ }^{[7]}$,其原理是在中性条件下,用过硫酸钾消解水样,将其中所含的多种含磷化合物全部氧化成正磷酸 盐,在酸性条件下正磷酸盐和钼酸铵、酒石酸锑钾反应形成磷钿杂多酸后,立即被抗坏血酸还原生成蓝色的 络合物, 可用比色法测定这种蓝色络合物的浓度 ${ }^{[8]}$. 锄酸铵分光光度法 (GB 11893-1989) 的检测下限为 $0.01 \mathrm{mg} / \mathrm{L}^{[9]}$, 但在水体中, 尤其是在我国南亚热带地区淡水水体中, 部分水体的总磷以及多数水体的溶解态

* 广东省水利科技创新项目(2012-10) 和水利部河湖健康评估广东省试点项目 (2013001) 联合资助. 2015-09-17 收稿;2016 - 02-28 收修改稿.黄丰明(1991 ), 男,硕士研究生;E-mail: hfm1122@ qq.com.

** 通信作者;E-mail: tljxiao@jnu.edu.cn. 
磷浓度均低于 $0.01 \mathrm{mg} / \mathrm{L}^{[10-12]}$. 鉴于目前大多数监测部门或研究机构多采用该方法检测水体中的磷浓度, 因 此, 明确该方法在监测低磷浓度水体可能出现的问题很有必要, 同时迫切需要对该方法进行合理有效的改 进, 提高对低磷浓度 $(<0.01 \mathrm{mg} / \mathrm{L})$ 水体检测的灵敏度和准确性.

从钿酸铵分光光度法的原理上分析, 可以从 3 个方面进行改进: 1)改变显色剂的配制方法 ${ }^{[13]}$, 减少显色 剂干扰; (2)改变测定波长, 提高灵敏度; (3)增加水样体积, 消解后不对水样进行稀释定容, 而是按消解前体积 定容. 通过对标准试样和野外水样的测定, 分析比较方法改进前后的灵敏度和准确性, 得到更适合检测淡水 水体尤其是南亚热带地区低磷浓度水体中磷的测定方法.

\section{1 材料与方法}

\section{1 仪器和设备}

自动蒸汽灭菌锅 (D-1 型) 用于总磷的氧化消解; 分光光度计 (Hitachi U-3900) 用于测定吸光度. $50 、 100$ $\mathrm{ml}$ 磨口比色管和 $3 \mathrm{~cm}$ 比色典.

\section{2 实验试剂}

实验过程中试剂用水为超纯水. 使用的化学药品包括: 抗坏血酸、锄酸铵、酒石酸锑钾和过硫酸钾, 均为 分析纯; 硫酸为优级纯.

实验试剂的配置: 1) 抗坏血酸溶液( $10 \%$ ) : 将 $10 \mathrm{~g}$ 抗坏血酸溶于水并稀释至 $100 \mathrm{ml}$;2) 抗坏血酸溶液 $(0.1 \mathrm{~mol} / \mathrm{L})$ : 将 $1.76 \mathrm{~g}$ 抗坏血酸溶于水并稀释至 $100 \mathrm{ml} ; 3)$ 钿酸盐溶液: 溶解 $13 \mathrm{~g}$ 钿酸铵于 $100 \mathrm{ml}$ 蒸馏水 中, 溶解 $0.35 \mathrm{~g}$ 酒石酸锑钾于 $100 \mathrm{ml}$ 蒸馏水中, 在不断摚拌下把钿酸铵溶液徐徐加到 $300 \mathrm{ml}$ 硫酸 $(1: 1)$ 中, 加酒石酸锑钾溶液并且混合均匀; 4) 酒石酸铇钾溶液: 将 $0.2743 \mathrm{~g}$ 酒石酸锑钾溶于水并稀释至 $100 \mathrm{ml}$; 5) 钿酸铵溶液 ( $4 \%$ ) : 将 $4 \mathrm{~g}$ 钿酸铵溶于水并稀释至 $100 \mathrm{ml}$; 6 ) 硫酸 $(14 \%)$ : 将 $14 \mathrm{ml}$ 浓硫酸稀释至 $100 \mathrm{ml}$; 7) 新显色剂: 将 $50 \mathrm{ml}$ 硫酸溶液 $(14 \%) 、 5 \mathrm{ml}$ 酒石酸锑钾溶液、15 $\mathrm{ml}$ 锄酸铵溶液 ( $4 \%$ ) 和 $30 \mathrm{ml}$ 抗坏血酸溶 液 $(0.1 \mathrm{~mol} / \mathrm{L}$ ) 混合而成, 现用现配; 8 ) 过硫酸钾溶液 ( $5 \%$ ) : 将 $5 \mathrm{~g}$ 过硫酸钾溶于水并稀释至 $100 \mathrm{ml}$; 9 ) 磷标 准溶液: 浓度为 $500 \mathrm{mg} / \mathrm{L}$, 由水利部水环境监测评价研究中心提供.

\section{3 实验方法}

1) 全波段扫描. 准确移取不同体积的磷标准溶液于一系列比色管中, 分别加人国标显色剂和新显色剂 后放置 $15 \mathrm{~min}$ 使其形成稳定的磷钿蓝络合物, 于波长 $450 \sim 1000 \mathrm{~nm}$ 间进行扫描, 寻找磷锄蓝的最大吸收峰.

2) 根据显色剂和测定波长的区别设置交叉实验, 得到以下 4 种比色法. $\mathrm{a}$ : 依次向 $50 \mathrm{ml}$ 水样中加人 $1 \mathrm{ml}$ 抗坏血酸溶液 $(10 \%)$ 和 $2 \mathrm{ml}$ 钿酸盐溶液, 上下倒转摇匀, 放置 $15 \mathrm{~min}$ 后于波长 $700 \mathrm{~nm}$ 处用 $3 \mathrm{~cm}$ 比色血测 定吸光度, 即国标法; $\mathrm{b}$ : 向 $50 \mathrm{ml}$ 水样中加人 $4 \mathrm{ml}$ 新显色剂, 上下倒转摇匀, 放置 $15 \mathrm{~min}$ 后于波长 $880 \mathrm{~nm}$ 处 用 $3 \mathrm{~cm}$ 比色㿼测定吸光度; $\mathrm{c}$ : 依次向 $50 \mathrm{ml}$ 水样中加人 $1 \mathrm{ml}$ 抗坏血酸溶液 $(10 \%)$ 和 $2 \mathrm{ml}$ 锄酸盐溶液, 上下 倒转摇匀, 放置 $15 \mathrm{~min}$ 后于波长 $880 \mathrm{~nm}$ 处用 $3 \mathrm{~cm}$ 比色典测定吸光度; $\mathrm{d}$ : 向 $50 \mathrm{ml}$ 水样中加人 $4 \mathrm{ml}$ 新显色 剂, 上下倒转摇匀,放置 $15 \mathrm{~min}$ 后于波长 $700 \mathrm{~nm}$ 处用 $3 \mathrm{~cm}$ 比色血测定吸光度.

对比在 4 种比色法下溶解态磷浓度的测定结果, 笁选最佳比色法, 测定水样中的总磷浓度.

3) 根据消解方式的不同, 以及上述笁选出的最佳比色法,设置 2 种总磷测定流程与方法.

M1: 水样的消解处理参考国标法 (GB 11893-1989), 即准确移取水样 $25 \mathrm{ml}$ 于 $50 \mathrm{ml}$ 比色管中, 向水样 中加人 $4 \mathrm{ml}$ 过硫酸钾溶液进行消解后, 定容至 $50 \mathrm{ml}$ 标线, 以下操作同上述的最佳比色法.

M2: 增加消解水样体积, 即准确移取水样 $50 \mathrm{ml}$ 于 $100 \mathrm{ml}$ 比色管中, 向水样中加人 $8 \mathrm{ml}$ 过硫酸钾溶液进 行消解后, 定容至 $58 \mathrm{ml}$, 再从经消解过的水样中另取出 $25 \mathrm{ml}$, 加人 $2 \mathrm{ml}$ 新显色剂, 以下操作同上述的最佳 比色法.

4) 标准曲线的绘制. 参照南亚热带湖库的实际磷浓度范围 ${ }^{[12]}$, 准确移取不同体积的磷标准溶液于一系 列比色管中, 以下操作同上述 2) 和 3) , 以磷浓度对其吸光度分别绘制溶解态磷和总磷标准曲线.

5) 加标回收实验. 设置 3 个梯度的低浓度磷标准试样: $0.0030 \pm 0.00016 、 0.0053 \pm 0.00027$ 和 $0.0083 \pm$ $0.00042 \mathrm{mg} / \mathrm{L}$, 分别用上述 4 种比色法及 M1 M2 法测定样品中的磷浓度, 每个样品做 6 个平行.

6) 水样总磷浓度的测定. 采集 3 份来自贫营养水库的水样, 另通过稀释得到 2 份更低浓度的水样, 以超 
纯水代替水样作空白,分别采用 M1、M2 法测定总磷浓度,每个样品做 6 个平行.

7)数据分析. 结果以平均值表示, 每组所得数据用 ANOVA 进行分析, 统计软件为 SPSS 18.0, 作图软件 为 Origin 8.0.

\section{2 结果与讨论}

\section{1 吸收波长和显色剂对磷测定的影响}

对磷锄蓝络合物进行全波段 (450 1000 nm) 扫描(图 1), 结果表明磷锄蓝有 2 个吸收峰, 分别为 $720 \mathrm{~nm}$ 和 $880 \mathrm{~nm}$ 波长位置, $880 \mathrm{~nm}$ 处的吸收峰要显著高于 $720 \mathrm{~nm}$ 处. 国标法中磷的测定波长为 $700 \mathrm{~nm}$, 考虑到我 国水质总磷浓度的测定标准是在 1989 年制定并开始实施的, 当时我国使用具有此波段范围分光光度计的 实验室不是很多, 如采用 $880 \mathrm{~nm}$ 作为测定标准在国内难以推广, 因此选择 $700 \mathrm{~nm}$ 测定波长的实用性更 强 ${ }^{[14]}$. 近 20 年来, 随着技术的发展, 分光光度计的稳定性和精确性更高, 分光光度计已能准确测定 $880 \mathrm{~nm}$ 处的吸光值. 在这种情况下, 选择 $880 \mathrm{~nm}$ 作为磷的测定波长更具优势, 尤其是对低磷浓度水体的磷测定, 其 灵敏度要比使用 $700 \mathrm{~nm}$ 作为测定波长更高.
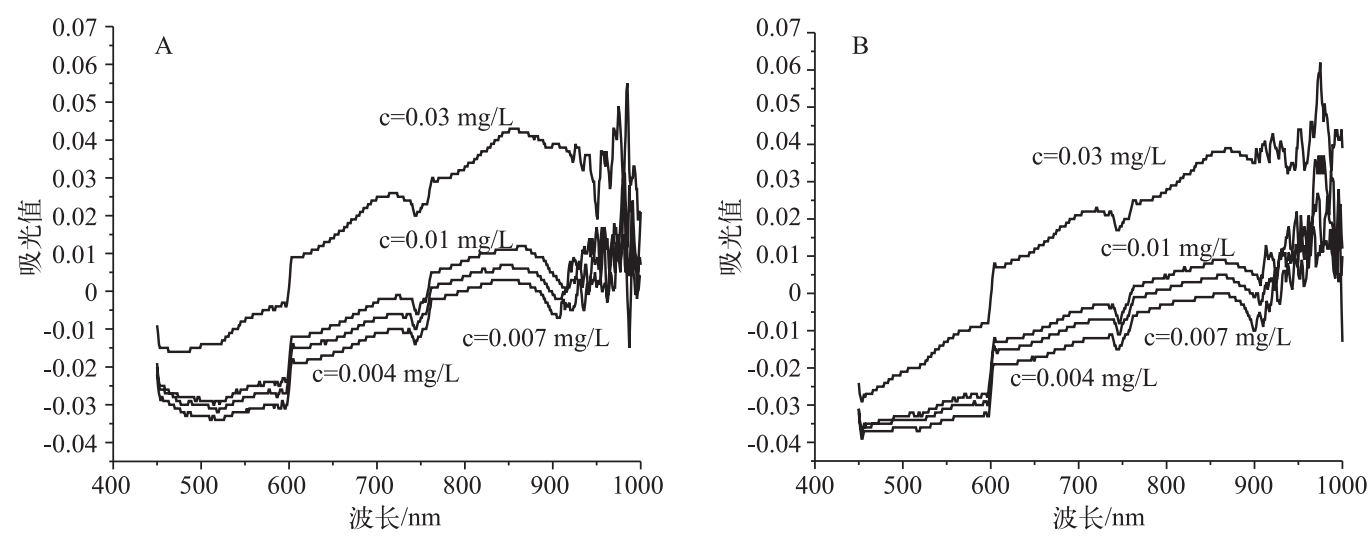

图 1 磷钼蓝全波段扫描图:A) 添加国标显色剂;B) 添加新显色剂

Fig. 1 Consecutive wavelength scanning of phosphorus molybdenum blue:

A) adding standard chromogenic agent; B) adding new chromogenic agent

对比相同磷浓度下添加 2 种显色剂试样的吸光值 (图 1), 结果表明国标显色剂的吸光值要高于新显色 剂. 添加国标显色剂的样品中钿酸铵浓度为 $0.98 \mathrm{~g} / \mathrm{L}$, 抗坏血酸浓度为 $1.89 \mathrm{~g} / \mathrm{L}$; 添加新显色剂的样品中锄 酸铵浓度为 $0.44 \mathrm{~g} / \mathrm{L}$, 抗坏血酸浓度为 $0.39 \mathrm{~g} / \mathrm{L}$. 在磷浓度测定中锄酸铵和抗坏血酸是显色剂的主要成分, 这两者在配制和添加过程中会产生一定的色度, 已有文献表明过多的钿酸根会形成其他不参与显色反应的 钿盐从而影响测定结果 ${ }^{[15]}$. 当样品中磷浓度较少时显色不明显 ${ }^{[16]}$, 显色剂本身的颜色会对测定造成一定干 扰. 新显色剂中各组分的浓度均低于国标显色剂,因此对吸光值的干扰要小于国标法.

\subsection{4 种方法对溶解态磷测定的差异}

2.2.1 4 种方法标准曲线的比较 对比 4 种方法在高磷浓度 ( >0.01 mg/L)下的标准曲线 (图 $2 \mathrm{~A}$ ), 4 种方法 的 $R^{2}$ 值均可达到 0.999 以上, 浓度和吸光值之间线性关系非常好, 均适用于高磷浓度水样的测定. 绘制 4 种 方法在低磷浓度 ( $<0.01 \mathrm{mg} / \mathrm{L}$ ) 下的标准曲线 (图 $2 \mathrm{~B}$ ), 结果表明方法 $\mathrm{b}$ 的 $R^{2}$ 值为 0.999 , 其线性关系相比于 其他 3 种方法更好.

方法 $b 、 c$ 所得标准曲线的斜率比方法 $a 、 d$ 所得标准曲线的斜率明显更大(图 2), 表明 $b 、 c$ 灵敏度更高, 方法的灵敏度和检出限有关, 灵敏度越高, 检出限越低 ${ }^{[17]}$, 因此方法 $b 、 c$ 的检出限要低于方法 $a 、 d$. 方法 $b 、 c$ 测定波长为 $880 \mathrm{~nm}$, 方法 $\mathrm{a} 、 \mathrm{~d}$ 的测定波长为 $700 \mathrm{~nm}$, 根据全波段扫描的结果, $880 \mathrm{~nm}$ 波长的峰值要高于 700 $\mathrm{nm}$ 的峰值, 因此方法 b、c 灵敏度的提高是由于 $880 \mathrm{~nm}$ 处吸收值更高导致的. 相对于 $700 \mathrm{~nm}, 880 \mathrm{~nm}$ 作为测 定波长时吸光值更高, 对不同浓度的区分更灵敏, 更适用于对低磷浓度水样的测定. 


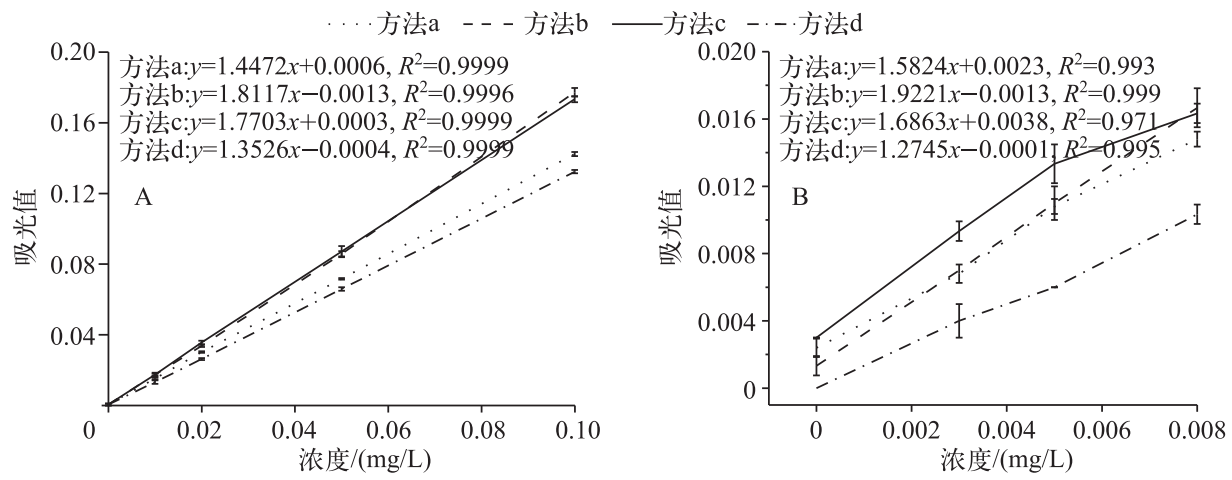

图 24 种方法的标准曲线和回归方程:A)高磷浓度;B)低磷浓度

Fig. 2 The standard curve and regression equation of four methods:

A) high phosphorus concentration; B) low phosphorus concentration

比较 4 种方法对空白样品的测定 (图 2B), 方法 $\mathrm{a} 、 \mathrm{c}$ 测定的吸光值明显大于方法 b、d. 空白值越大, 将导 致最小分析信号升高 ${ }^{[18]}$, 而随着被测组分浓度的下降, 空白值对测定结果准确性的影响也越来越大 ${ }^{[19]}$. 方 法 $a 、 c$ 添加的是国标显色剂,方法 $b 、 d$ 添加的是新显色剂,这与全波段扫描所得结果一致, 可见新显色剂可 以减少对低磷浓度样品测定时的干扰,更适用于对低磷浓度水样的测定.

综合以上分析, 方法 $\mathrm{b}$ 的灵敏度更高和空白值更小, 相对其他 3 种方法, 更适用于对低磷浓度水样的 测定.

\subsubsection{4 种方法对低浓度溶解态磷标样测定结}

果的比较 加标回收实验是化学分析中重要 的质控手段, 回收率是判定结果准确度的量 化指标 ${ }^{[20]}$. 实验结果表明 (图 3), 方法 $\mathrm{b}$ 的回 收率与其它 3 种方法差异极显著 $(F=17.86$, $P<0.01)$, 且平均值最趋近于 $100 \%$, 故相对于 其他 3 种方法, 方法 $\mathrm{b}$ 具有较高的准确性, 更 适用于对低磷浓度水样的测定.

加标回收结果表明, 方法 b 能够准确测 定 $0.003 \mathrm{mg} / \mathrm{L}$ 以上的浓度, 因此本实验确定 方法 $\mathrm{b}$ 的最低检测限可达到 $0.003 \mathrm{mg} / \mathrm{L}$. 鉴 于 $0.01 \mathrm{mg} / \mathrm{L}$ 只对于部分浮游植物是磷限制 性磷浓度, 而 $0.003 \mathrm{mg} / \mathrm{L}$ 对于绝大多数浮游 植物是磷限制性磷浓度 ${ }^{[5]}$, 因此溶解态磷的 检测下限扩展到 $0.003 \mathrm{mg} / \mathrm{L}$, 对于浮游植物 群落生态学的研究有重要意义.

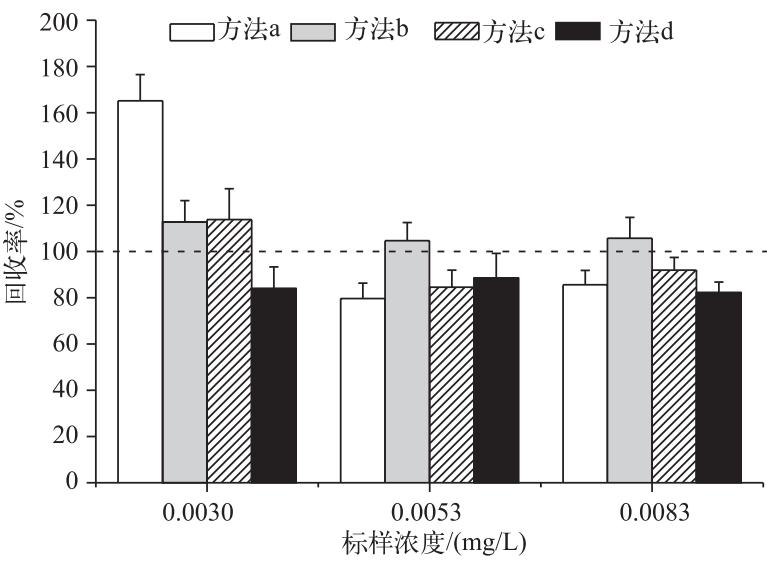

图 34 种比色法测定低磷浓度标样的回收率

Fig.3 The recovery ratio of low phosphorus concentration standard sample determined by four methods

在夏季, 广东省多数大型水库出现稳定 水体分层, 上层水体的溶解态磷浓度远低于 $0.01 \mathrm{mg} / \mathrm{L}$, 例如流溪河水库、新丰江水库、高州水库夏季的溶解 态磷浓度均在 $0.005 \mathrm{mg} / \mathrm{L}$ 以下 ${ }^{[21]}$, 在这种情况下,使用方法 $\mathrm{b}$ 进行磷浓度测定更好.

\section{$2.3 \mathrm{M} 1 、 \mathrm{M} 2$ 法对总磷浓度测定的差异}

2.3.1 M1、M2 法标准曲线的比较 对比 M1、M2 法在高磷浓度 $(>0.01 \mathrm{mg} / \mathrm{L}$ ) 下的标准曲线 (图 $4 \mathrm{~A})$, 其 $R^{2}$ 值 均可达到 0.999 以上, 适用于高磷浓度水样的测定. 比较 M1、M2 法在低磷浓度 $(<0.01 \mathrm{mg} / \mathrm{L})$ 下的标准曲线 (图 4B), M2 法的 $R^{2}$ 值为 0.999 , 浓度和吸光值之间线性关系较好; M1 法的 $R^{2}$ 值为 0.928 , 其线性关系较差.

M1 法消解后需对水样进行二次稀释定容, 即将 $25 \mathrm{ml}$ 水样定容到 $50 \mathrm{ml}$ 后再进行比色分析, 而 M2 法消 
解后不对水样进行稀释定容, 而是按消解前体积定容后比色, 因此 M1 法的检测下限相对 M2 法要高. 按照 方法 b 的检测下限, M2 法的检测下限仍然为 $0.003 \mathrm{mg} / \mathrm{L}$, 但 M1 法的检测下限提高到 $0.006 \mathrm{mg} / \mathrm{L}$, 对低于该 限值的水样, 将无法保证结果的准确性. 实验结果也表明, M1 法在浓度为 0.003 和 $0.005 \mathrm{mg} / \mathrm{L}$ 处吸光值无 明显差异.

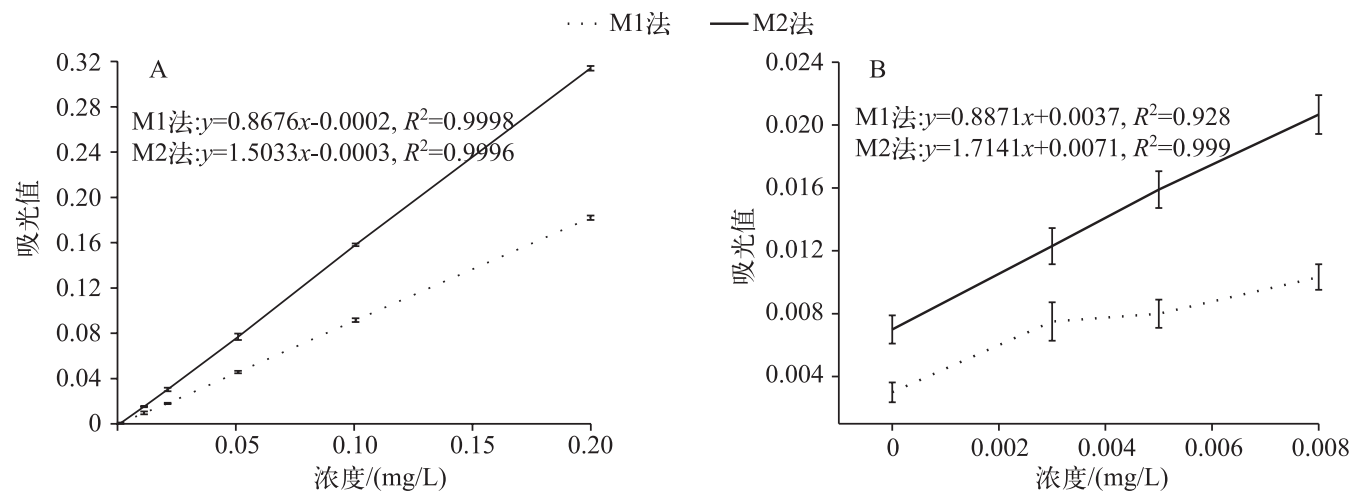

图 4 总磷标准曲线和回归方程:A) 高磷浓度;B)低磷浓度

Fig.4 The standard curve and regression equation of total phosphorus:

A) high phosphorus concentration; B) low phosphorus concentration

2.3.2 M1、M2 法对低浓度总磷标样和野外水样测定结果的比较 低浓度总磷标样的加标回收实验结果表明 (图 5A), 相对于 M1 法, M2 法测定的回收率变化较小, 且更趋近于 $100 \%$, 因此 M2 法的准确性和稳定 性更高.

用 M1、M2 法分别测定 5 份野外水样中的总磷浓度 (图 5B), 表明 2 种方法测定的水样总磷浓度差异不 显著 $(P>0.05)$, 但 M1 法测得的总磷浓度总体上均高于 M2 法. M1 法测定低磷浓度水样时, 磷浓度和吸光值 间线性相关性较差, 会导致绘制的标准曲线斜率出现偏差, 本实验中表现为标准曲线斜率偏小 (图 4B), 因 此用该标准曲线计算出来的浓度会比实际浓度偏大,这也是 M1 法测得的浓度高于 M2 法的原因. 反之, 若 绘制的标准曲线斜率偏大, 则计算出来的浓度则会偏低. 可见 M1 法由于消解后需要稀释定容, 进一步降低 了低磷浓度样品中的磷浓度而使分光光度计对浓度差异的响应不灵敏, 导致浓度和吸光值间的线性关系较 差和标准曲线绘制出现偏差,使最终测得的结果无法真实反映水样的实际浓度.
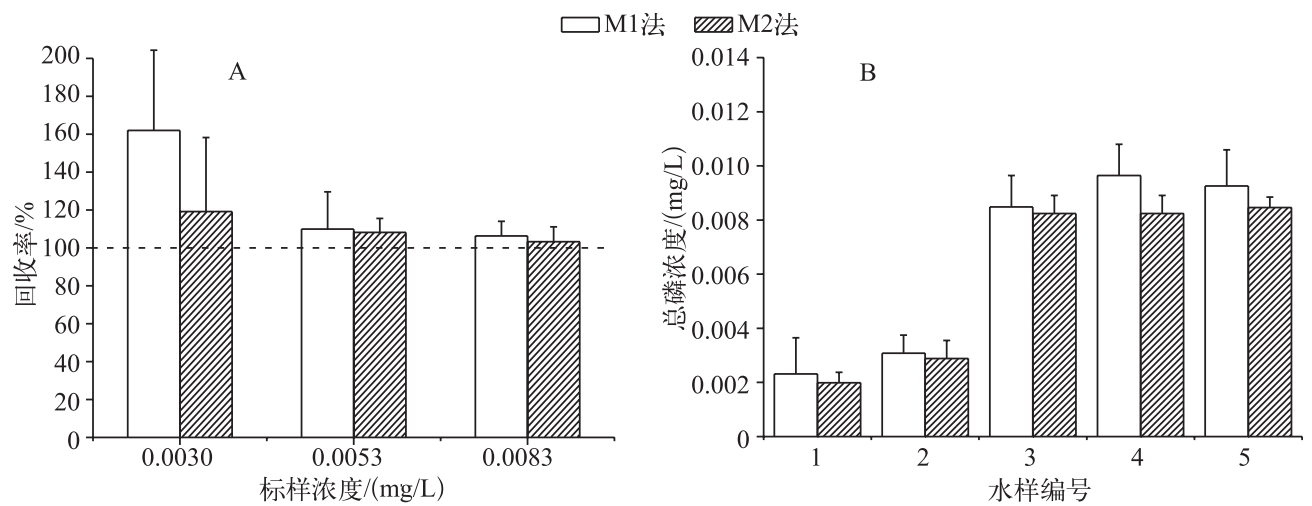

图 5 M1 和 M2 法测定低浓度总磷标样的回收率 $(\mathrm{A})$ 和野外水样中的总磷浓度 $(\mathrm{B})$

Fig.5 The recovery ratio of low total phosphorus concentration standard sample(A) and total phosphorus concentration of field sample(B) determined by M1 and M2 
综上以上分析, M2 法相对于 M1 法更适用于对低磷浓度水样的测定.

\section{3 结论}

对于低磷浓度水体 (总磷浓度低于 $0.01 \mathrm{mg} / \mathrm{L}$ ), 现行的国标磷测定法 ( GB 11893-1989) 准确性和灵敏 度均较差. 可从 3 个方面进行改进提高测定的准确性: 1) 增加消解水样体积至 $50 \mathrm{ml}$, 消解后不再进行二次 稀释定容 (即 M2 法) ; 2 ) 改变显色剂的配制 (即 $14 \%$ 硫酸溶液 $50 \mathrm{ml}$ 、酒石酸锑钾溶液 $5 \mathrm{ml} 、 4 \%$ 钼酸铵溶液 $15 \mathrm{ml}$ 和 $0.1 \mathrm{~mol} / \mathrm{L}$ 的抗坏血酸溶液 $30 \mathrm{ml}$ 混合而成, 现配现用), 减少显色剂干扰; 3 ) 将测定波长设置为 880 $\mathrm{nm}$, 提高灵敏度. 通过改进后, 新方法能准确有效测定磷浓度在 $0.003 \sim 0.01 \mathrm{mg} / \mathrm{L}$ 范围内的水样.

\section{4 参考文献}

[ 1 ] Conley DJ, Paerl HW, Howarth RW et al. Controlling eutrophication: nitrogen and phosphorus. Science, 2009, 323 (5917) : 1014-1015. DOI: 10.1126/science.1167755.

[ 2 ] Kalff J. Limnology: inland water ecosystems. New Jersey: Prentice Hall, 2002.

[ 3 ] Abell JM, Özkundakci D, Hamilton DP. Nitrogen and phosphorus limitation of phytoplankton growth in New Zealand lakes: implications for eutrophication control. Ecosystems, 2010, 13(7) : 966-977. DOI: 10.1007/s10021-010-9367-9.

[ 4 ] Elser JJ, Bracken MES, Cleland EE et al. Global analysis of nitrogen and phosphorus limitation of primary producers in freshwater, marine and terrestrial ecosystems. Ecology Letters, 2007, 10(12) : 1135-1142. DOI: 10.1111/j. 1461-0248. 2007.01113.x.

[ 5 ] Reynolds CS. The ecology of phytoplankton. Cambridge: Cambridge University Press, 2006.

[ 6 ] Sun Jianmin, Cui Meng, Gao Zheng. Study and proceeding on the determination of trace phosphorus. Studies of Trace Elements and Health, 2005, 22(2) : 55-58( in Chinese with English abstract). [ 孙建民, 崔萌, 高峥. 痕量磷测定方法的 研究及新进展. 微量元素与健康研究, 2005, 22(2): 55-58.]

[ 7 ] Peng Ganghua, Kang Changan, Zhong Hongyan et al. Quality control index of total phosphorus test in water sample using ammonium molybdate spectrophotometric method. Environment and Ecology in the Three Gorges, 2011, 33(2) : 31-34(in Chinese with English abstract). [彭刚华, 康长安, 钟鸿雁等. 钼酸铵分光光度法测定水中总磷质量控制指标研究. 三峡环境与生态, 2011, 33(2) : 31-34.]

[ 8 ] China Environmental Protection Administration, Editorial Board of Water and Wastewater Monitoring and Analysis Method. Water and wastewater monitoring and analysis method: The fourth edition. Beijing: China Environmental Science Press, 2002 (in Chinese). [ 国家环境保护总局《水和废水监测分析方法》编委会. 水和废水监测分析方法: 第 4 版. 北京: 中国环境科学出版社, 2002.]

[ 9 ] GB 11893-1989. Water quality—determination of total phosphorus-ammonium molybdate spectrophotometric method. Beijing: China Standard Press, 1989 (in Chinese). [ GB 11893-1989. 水质总磷的测定一一钼酸铵分光光度法. 北 京: 中国标准出版社, 1989.]

[10] Xu Ning, Duan Shunshan, Lin Qiuqi et al. Analysis on phosphorus pollution and eutrophication of the large reservoirs for water supply in Guangdong Province. Ecologic Science, 2003, 22(4) : 341-345( in Chinese with English abstract). [徐 宁, 段舜山, 林秋奇等. 广东大中型供水水库的磷污染与富营养化分析. 生态科学, 2003, 22(4): 341-345. ]

[11] Lin Qiuqi, Hu Ren, Duan Shunshan et al. Reservoir trophic states and the response of plankton in Guangdong Province. Acta Ecologica Sinica, 2003, 23(6): 1101-1108(in Chinese with English abstract). [林秋奇, 胡韧, 段舜山等. 广东 省大中型供水水库营养现状及浮游生物的响应. 生态学报, 2003, 23(6) : 1101-1108.]

[12] Jiang Tao, Liu Zufa, Chen Xiaohong et al. Assessment of reservoir eutrophication in Guangdong Province. J Lake Sci, 2005, 17(4) : 378-382 (in Chinese with English abstract). DOI: 10.18307/2005.0417. [ 江涛, 刘祖发, 陈晓宏等. 广 东省水库富营养化评价. 湖泊科学, 2005, 17(4) : 378-382.]

[13] U.S. Environmental Protection Agency. Methods of chemical analysis of water and wastes. USEPA Rep. 600/4-79-020. USEPA, Cincinnati, OH. 1979.

[14] Zhang Hui. Study on colorimetry wavelength choice of phosphorus molybdenum blue. Agro-environmental Protection, 1991, 10(6) : 274,277 (in Chinese with English abstract). [张辉. 磷的钼蓝比色波长选择的研究. 农业环境保护, 1991, 10 (6) : $274,277$. 
[15] Tsoulfanidis IA, Tsogas GZ, Giokas DL et al. Design of a field flow system for the on-line spectrophotometric determination of phosphate, nitrite and nitrate in natural water and wastewater. Microchimica Acta, 2008, 160(4) : 461-469. DOI: 10. 1007/s00604-007-0789-9.

[16] Huang Cheng, Zhou Yan, Zeng Ganning et al. Determination of phosphorus in seaweed by molybdenum blue spectrophotometry with ascorbic acid reducing agent. Marine Environmental Science, 2009, 28 (A01): 76-78 (in Chinese with English abstract). [黄城, 周燕, 曾淦宁等. 抗坏血酸-钼蓝分光光度法测定海藻中磷的研究探讨. 海洋环境科学, $2009, \mathbf{2 8}(\mathrm{z} 1):$ 76-78.]

[17] Cha Dongmei. The elementary discussion of sensitivity, detection limit and determination limit. University Chemistry, 2011, 26(4) : 84-86 (in Chinese with English abstract). [察冬梅. 浅议灵敏度, 检出限和测定限. 大学化学, 2011, 26(4) : 84-86.]

[18] Jiang Zigang. The analysis margin in trace analysis. Shanghai Environmental Sciences, 1987, (1): 8(in Chinese with English abstract). [蒋子刚. 痕量分析中的分析空白. 上海环境科学, 1987,(1):8.]

[19] Xu Jirong. The discussion on the blank value of the sample in marine environmental monitoring. Marine Environmental Science, 1996, 15(2): 12-16( in Chinese with English abstract). [ 徐继荣. 海洋环境监测中有关分析空白值的探讨. 海 洋环境科学, 1996, 15(2): 12-16.]

[20] Ren Chengzhong, Mao Lifen. Study on the practice of standard recovery test and calculation of recovery rate. Industrial Safety and Environmental Protection, 2006, 32(2) : 9-11 (in Chinese with English abstract). [任成忠, 毛丽芬. 加标回 收实验的实施及回收率计算的研究. 工业安全与环保, 2006, 32(2) : 9-11.]

[21] Xie Difei. Eutrophication evaluation of large and medium reservoirs in Guangdong Province and design of water quality management information system[Dissertation]. Guangzhou: Jinan University, 2002(in Chinese with English abstract). [谢 涤非. 广东省大中型水库富营养化评价及水质管理信息系统研制 [ 学位论文]. 广州: 暨南大学, 2002.] 